\title{
Prevalencia de hepatitis B y C en donadores de sangre de un hospital privado
}

\author{
Prevalence of hepatitis B and C in private hospital blood donors \\ María José Ortega Chavarría, * Silvia Nayeli Ahumada Zavala, \\ Enrique Díaz Greene, ${ }^{\S}$ Federico Rodríguez Weber ${ }^{\S}$
}

\section{Resumen}

La infección por virus de hepatitis B (VHB) y C (VHC) es un problema de salud pública mundial y un riesgo grave para la medicina transfusional, pues es una causa importante de daño hepático en México. Objetivo: Determinar la prevalencia de VHB y VHC en donadores de sangre que acudieron al Hospital Ángeles Pedregal en un lapso de 19 meses. Material y métodos: Estudio descriptivo y retrospectivo, realizado del 1 de enero de 2018 al 31 de julio de 2019 en el que se incluyeron a 5,705 donadores. Resultados: De los 4,030 casos seleccionados, $30.72 \%$ correspondió a mujeres y $69.28 \%$ a hombres; se identificaron 22 donaciones altruistas y 4,008 para reposición de hemoderivados. La seroprevalencia global observada en nuestra población fue de $0.27 \%$ para VHC y $0.04 \%$ para VHB. La infección por VHC tuvo mayor frecuencia en el sexo femenino entre los 45 a 65 años con tres casos. Conclusiones: Se encontró una baja prevalencia de infección por VHB y VHC, la cual fue de 0.27 y $0.04 \%$ respectivamente, y en nuestra población hubo 5.5 veces más prevalencia de la infección por VHC que por VHB. Probablemente se debió a que la población que acude a donar sangre se encuentra generalmente sana.

Palabras clave: Hepatitis B, hepatitis C, banco de sangre, transfusión.

\section{INTRODUCCIÓN}

Se sabe que en 1666 Richard Lower realizó la primera transfusión sanguínea entre animales, y para 1667 el médico francés Jean-Baptiste Denys llevó a cabo la primera transfusión sanguínea entre humanos. En 1900, Karl

* Residente del Curso de Medicina Interna. Facultad Mexicana de Medicina de la Universidad La Salle, México.

₹ Residente del Curso de Patología Clínica. Facultad de Medicina de la Universidad Autónoma de México, Ciudad de México.

$\S$ Profesor del Curso de Medicina Interna. Facultad Mexicana de Medicina de la Universidad La Salle, México.

Hospital Ángeles Pedregal.

\section{Abstract}

Infections caused by hepatitis B virus (HBV) and hepatitis $C$ virus (HCV), are global health issues and a serious risk associated to transfusional medicine; especially being one of the most important causes of liver damage in Mexico. Objective: To determine the prevalence of HBV and HCV in blood donors in Hospital Angeles Pedregal in a time lapse of 19 months. Material and methods: Retrospective and descriptive study carried out between the 1st of January 2018 and the 31st of July 2019 , which included 5,705 donors. Results: Of 4,030 selected cases, $30.72 \%$ were female and $69.28 \%$ were male. We identified 22 altruistic donors and 4,008 for replacement of blood products. Global seroprevalence observed in our population was $0.27 \%$ for $\mathrm{HCV}$ and $0.04 \%$ for HBV. HVC infection was more frequent in females between 45 to 65 years, with three cases. Conclusions: A low prevalence of HBV and HVC infection was found, being $0.27 \%$ and $0.04 \%$, respectively, $\mathrm{HCV}$ prevalence was found to be 5.5 times greater than HBV. These findings are probably explained because blood donors generally are healthy.

Keywords: Hepatitis B, hepatitis C, blood bank, transfusion.

Landsteiner descubrió los grupos sanguíneos $\mathrm{ABO}$ y, en esa misma década, Hektoen habló de la necesidad de realizar "pruebas sanguíneas cruzadas" con el fin de evitar reacciones transfusionales. En 1935 se creó el primer banco de sangre en la Clínica Mayo y durante la Segunda Guerra Mundial se obtuvieron grandes avances, y asimismo fue

Correspondencia:

Dr. Federico Rodríguez Weber,

Correo electrónico: fweber@saludangeles.com

Aceptado: 26-09-2019.

www.medigraphic.com/actamedica

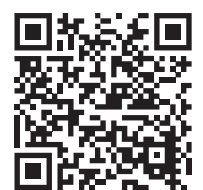


reportada la hepatitis postransfusional. ${ }^{1}$ El riesgo residual de las infecciones asociadas con la transfusión sanguínea ha disminuido en los países que han implementado tamizajes serológicos de forma rutinaria para los donadores. ${ }^{2}$

La infección por VHB y VHC es un problema de salud pública a nivel mundial, pues es causa importante de hepatitis crónica, cirrosis y carcinoma hepatocelular. Alrededor de 257 millones cursan con infección crónica por VHB y 71 millones con VHC. La historia natural depende de la edad de infección, forma de adquisición, etnia y genotipo. ${ }^{3,4} \mathrm{La}$ distribución es mundial, aunque su prevalencia varía dependiendo de cada país; actualmente se considera endémico para VHB el Sureste Asiático, China, África subsahariana y la India, donde la mayoría de las infecciones se producen durante la infancia. ${ }^{5}$ Entre las principales vías de transmisión asociadas con ambos virus destacan: transfusión sanguínea (antes de 1992), consumo de drogas por vía parenteral o no parenteral, tatuajes y perforaciones realizadas en condiciones de higiene deficiente, transmisión perinatal sobre todo para el VHB (países en vías de desarrollo), transmisión sexual (menos común para el $\mathrm{VHC}$ ) y, por último, la transmisión nosocomial. ${ }^{6}$

El VHB es un virus DNA pequeño de la familia Hepadnaviridae, el cual fue descubierto en 1963 por el médico Baruch S. Blumberg. Se sabe que hasta 30\% de la población mundial muestra evidencia serológica de una infección previa, si se tiene en cuenta que su prevalencia e incidencia no siguen un perfil uniforme de endemicidad. ${ }^{7}$ Los marcadores serológicos con los que contamos actualmente son: $\mathrm{HBsAg}$ y anti-HBs, HBeAg y anti-HBe, y anti-HBc IgM e $\operatorname{lgG} ;{ }^{8}$ de éstos, los geno $\mathrm{H}$ y $\mathrm{G}$ son los que predominan en México. ${ }^{9}$ La presencia de HBsAg y anti-HBc positivos es un indicador de infección por VHB; en tanto el resultado de anti-HBC positivo de forma aislada debe hacernos pensar

Figura 1: Distribución de la población estudiada.

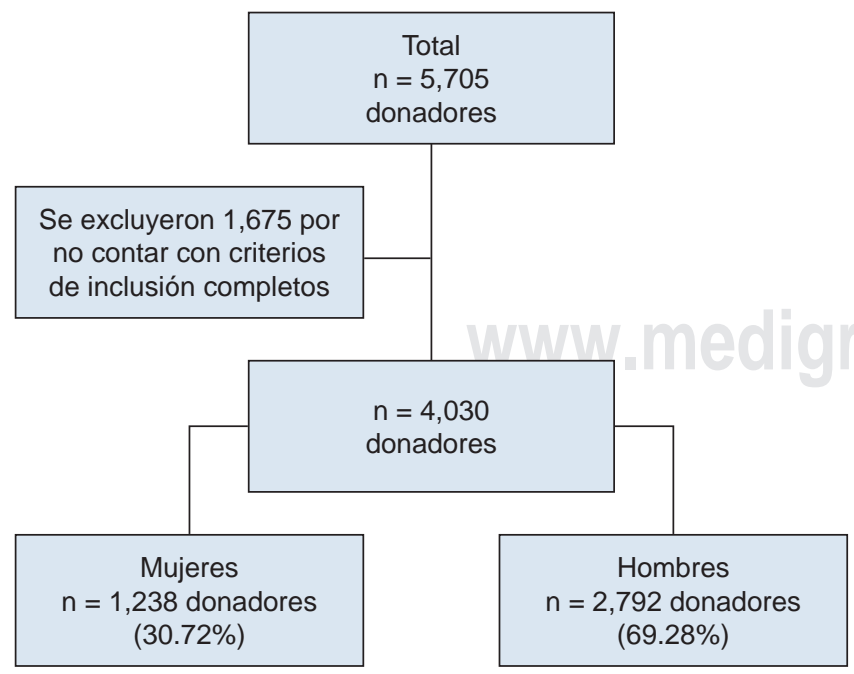

Figura 2: Concentrado de población.

Población de donadores

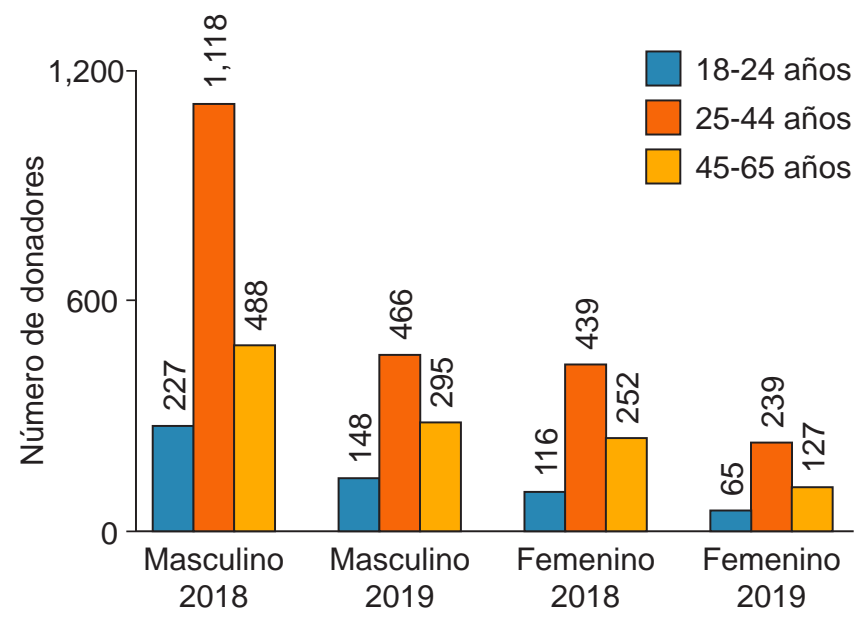

en un periodo de ventana o una infección resuelta, pues son necesarios estudios adicionales para aclarar esta situación. La vacuna tiene una eficacia de 95\% en la prevención de esta misma infección. ${ }^{10}$

El VHC es un virus RNA pequeño de la familia Flaviviridae con descripción de 6 genotipos y más de 50 subtipos y representa el patógeno de transmisión sanguínea más frecuente a nivel mundial; ${ }^{11}$ aunque en México el genotipo que predomina es el $1 \mathrm{~b}$, hay una menor proporción de $1 \mathrm{a}$, 2a y $3 \mathrm{~b} .{ }^{12}$ Actualmente, se cuenta con terapias de cursos entre ocho a 16 semanas que muestran eficacia de hasta $90 \% .{ }^{13}$ A diferencia del VHB, no se cuenta con una vacuna preventiva contra $\mathrm{VHC}$.

El diagnóstico molecular ha permitido la identificación directa de los virus, así como la determinación de genotipos y subtipos virales, siendo una parte esencial de la práctica médica para el manejo y tratamiento de los pacientes. ${ }^{14} \mathrm{~A}$ pesar de lo anterior, el riesgo de transmisión persiste en los bancos de sangre, debido a la ventana inmunológica, a los donantes asintomáticos como portadores crónicos, a la infección con cepas mutantes o a los errores técnicos. ${ }^{15} \mathrm{~A}$ pesar de las nuevas regulaciones para los bancos de sangre, la norma no específica que se debe realizar con metodologías de inmunoenzimática y/o inmunoensayo por quimioluminiscencias, las cuales aportan una sensibilidad de $99.5 \%$ y especificidad de $99 \%$.

Así, el objetivo de este estudio es determinar la frecuencia de $\mathrm{VHB}$ y $\mathrm{VHC}$ en donadores de sangre que acudieron al Hospital Ángeles Pedregal en un periodo de 19 meses.

\section{MATERIAL Y MÉTODOS}

Se trata de un estudio descriptivo y retrospectivo. Se empleó una fuente de información secundaria bajo completa 
confidencialidad. Éste se llevó a cabo en individuos que acudieron a donar sangre del 1 de enero de 2018 al 31 de julio del 2019 al Banco de Sangre del Hospital Ángeles Pedregal, Ciudad de México. Se tomaron en cuenta los criterios de inclusión y exclusión dispuestos por la Norma Oficial Mexicana NOM-253-SSA1-2012 para la disposición de sangre humana y sus componentes con fines terapéuticos. Se contó con una población inicial de 5,705 pacientes, pero fueron excluidos 1,675 casos por contar con algún criterio de exclusión, por lo que al final obtuvimos 4,030 casos como población final.

Criterios de inclusión. Se tomaron en cuenta a los individuos entre 18 a 65 años, sin importar el sexo y que contaran con una valoración previa por el personal calificado.

Criterios de exclusión. Pacientes menores de 18 años o mayores de 65 años, mujeres embarazadas, pacientes con enfermedades crónicas y/o infecciosas activas, conductas sexuales de riesgo, toxicomanías, dieta ovolactovegetariana o vegana, consumo de productos herbolarios o tratamiento farmacológico crónico, así como todos aquellos pacientes que no contaran con los niveles séricos de hemoglobina, hematocrito y número total de leucocitos marcados por la norma.

De acuerdo con la Norma Oficial Mexicana NOM253-SSA1-2012, se deben realizar pruebas obligatorias a todas las unidades obtenidas para donación que permitan la búsqueda de los siguientes agentes infecciosos: a) Treponema pallidum, b) virus B de la hepatitis, c) virus $C$ de la hepatitis, d) virus de la inmunodeficiencia humana tipos 1 y 2, y e) Trypanosoma cruzi. En nuestra institución se utilizan inmunoensayos por micropartículas quimioluminiscentes dirigidos a los antígenos específicos de cada agente infeccioso; para ello se emplea el equipo ARCHITECT i1000SR Abbott. Los reactivos que utiliza el equipo son de tercera generación, por lo que en el caso de tamizaje de hepatitis B se busca el antígeno de superficie del virus de la hepatitis B (antígeno Australia) y para la hepatitis $C$ es la búsqueda de anticuerpos contra el virus de hepatitis $C$.

\section{Análisis estadístico}

En este estudio se analizaron a todos los donantes que acudieron al Banco de Sangre del Hospital Ángeles Pedregal en el periodo de tiempo establecido, los cuales fueron clasificados por grupo etario y por sexo. Se realizaron las pruebas obligatorias dictadas por la Norma Oficial Mexicana NOM-253-SSA1-2012: para la disposición de sangre humana y sus componentes con fines terapéuticos, y posterior a esto se identificó la frecuencia para VHB y VHC.

\section{RESULTADOS}

De un total de 5,705 donadores, únicamente se tomaron los casos aptos para donación con una población final de 4,030 casos. El rango de edad fue de entre 18 a 65 años, de los cuales 1,238 eran mujeres (30.72\%) y 2,792 hombres (69.28\%) con una proporción 2:1 (hombre:mujer). Del total de los donadores 4,008 fueron para reposición familiar y 22 casos altruistas (Figura 1).

En la Figura 2 se concentra el número total de donadores con el número respectivo por año, sexo y edad.

Se encontró un mayor número de donadores del sexo masculino de entre 25-44 años, con un total de 1,584 individuos, lo que equivale a $39.3 \%$ de la población total, seguidos del grupo etario de 45-65 años, con un total de $783(28 \%)$. Dentro del grupo de las mujeres, la menor frecuencia se encontró en el grupo de entre 18-24 años, pues hay un mayor número de donantes en el grupo de 25-44 años con 678 casos (54.7\%). No se identificó un patrón específico de donación a través del tiempo.

La seropositividad encontrada fue de dos casos de hepatitis B y 11 casos positivos de hepatitis C (Tabla 1). De los casos de hepatitis $\mathrm{C}$, sólo dos fueron confirmados por inmunoblot y uno de ellos contaba con antecedentes de infección (múltiples tatuajes); el resto de los donadores con serología reactiva no se presentaron a estudios confirmatorios ni se encontraron con antecedentes de riesgo asociados con infección por VHB o VHC. La seroprevalencia global observada en nuestra po-

\begin{tabular}{|c|c|c|c|c|c|c|c|}
\hline \multirow[b]{2}{*}{ Edad } & \multicolumn{3}{|c|}{ Femenino } & \multicolumn{3}{|c|}{ Masculino } & \multirow[b]{2}{*}{ Total } \\
\hline & $18-24$ & $25-44$ & $45-65$ & $18-24$ & $25-44$ & $45-65$ & \\
\hline VHB & 0 & 1 & 0 & 0 & 0 & 1 & 2 \\
\hline $\mathrm{VHC}$ & 2 & 1 & 3 & 1 & 2 & 2 & 11 \\
\hline
\end{tabular}


blación fue de $0.27 \%$ para hepatitis C, siendo de $0.15 \%$ en mujeres y $0.12 \%$ en hombre; en tanto para hepatitis B fue de $0.04 \%$ sin diferencia por género. La frecuencia fue 5.5 veces mayor para $\mathrm{VHC}$ que para $\mathrm{VHB}$. Ninguno de los casos de donación altruista presentó serología positiva.

De las pruebas obligatorias por norma oficial, durante el lapso estudiado, se encontró que el VHC fue el que tuvo la mayor prevalencia, con un total de $11 / 29$ casos $(37.93 \%$ ) y VHB con presencia de 2/29 casos (6.9\%) (Tabla 2).

\section{DISCUSIÓN}

La prevalencia en nuestro país varía considerablemente dependiendo de la zona geográfica estudiada; así, es posible encontrar mayor positividad en los Estados cercanos a ambas fronteras. La Organización Mundial de la Salud (OMS), en su último reporte, marca la prevalencia de $\mathrm{VHB}$ y $\mathrm{VHC}$ en 0.7 y $1 \%$ respectivamente para las Américas. ${ }^{16}$ En México, los nuevos casos por cada 100 mil habitantes se han mantenido estables entre los años 2013 y 2015, tanto para la hepatitis B como C, con alrededor de 0.6 y 1.7-1.8 casos/100 mil habitantes respectivamente; estas cifras nos hablan de la población en general sin tomar en cuenta los portadores asintomáticos. ${ }^{17}$

La detección de la infección viral por inmunoanálisis de micropartículas quimioluminiscentes para la detección de antígenos o anticuerpos, empleados por ARCHITECT i1000SR Abbott, ${ }^{18}$ muestra una sensibilidad de $98.7 \%$ y una sensibilidad de $96.4 \%$ para $\mathrm{VHC} ;{ }^{19}$ una sensibilidad de 98\% con especificidad de $95 \%$ para $\mathrm{HBsAg}^{20}$ y para anti$\mathrm{HBC}$ la sensibilidad es de $99.1 \%$ y la especificidad es mayor a $99.5 \% .{ }^{21}$ Esto demuestra una baja posibilidad de falsos negativos y/o positivos y una buena validez en la estimación de la prevalencia de infección. Aunado a esto, como parte de la vigilancia de los bancos en México, el Centro Nacional de la Transfusión realiza un control de calidad externo al Banco del Hospital Ángeles Pedregal para poder constatar datos fidedignos sobre la serología que se le examina a cada donador. El análisis de los donadores en un hospital de tercer nivel permite un mejor control de la calidad de la sangre y la identificación de factores infecciosos de relevancia; también lo permite una mejor evaluación de los donadores para disminuir el riesgo de infección residual durante las transfusiones, teniendo datos de una prevalencia para VHC de hasta $0.77 \%$ en la Ciudad de México ${ }^{22}$ y una proporción que oscila entre 0.16 a $0.32 \%$ para $\mathrm{VHB} .^{23}$

Méndez-Sánchez y colaboradores publicaron un estudio transversal en el que se reportó una prevalencia de $\mathrm{VHC}$ de 0.47 y $0.11 \%$ para VHB de su población total $(9,099$ donadores) y se trató de un estudio en un hospital privado. ${ }^{24}$ En este estudio se evidenció que el grupo etario de 25-44 años en ambos sexos fue el que más acudió a donar sangre. En lo general, predominó la causa por reposición (familiar) y únicamente 22 donaciones fueron altruistas; además, aportó referencias sobre el comportamiento de infecciones en la población del banco de sangre.

El predominio de la donación en hombres puede ser un reflejo de que las mujeres sufren más eventos de anemia, posiblemente secundaria al sangrado transvaginal u otras causas de ésta (malos hábitos alimenticios), así como embarazo, bajo peso, venas delgadas, entre otros.

La seroprevalencia global observada en nuestra población fue de $0.27 \%$ para hepatitis C y para hepatitis B fue de $0.04 \%$. Se encontró que la frecuencia para $\mathrm{VHC}$ fue 5.5 veces mayor que para $\mathrm{VHB}$; la infección por $\mathrm{VHC}$ se mostró heterogénea, afectando a ambos sexos y teniendo mayor frecuencia entre 45 a 65 años en el sexo femenino con tres casos. En el caso de VHB no se encontró una prevalencia ni para el sexo ni para la edad. Es de relevancia que ninguno de los casos de donación altruista contó con pruebas reactivas, aunque también, al ser un porcentaje pequeño $(0.5 \%)$ del total de donadores, no es valorable como en el resto de los donadores de reposición.

La baja proporción de donaciones altruistas deja en descubierto una pobre concientización de la población o una baja implementación de campañas informativas sobre donación; así, en el año 2015 se registraron únicamente 82,365 donaciones de forma altruista. ${ }^{25}$ Haciendo un comparativo con los estudios de prevalencia en donadores de sangre de nuestro país, se reportan cifras similares que tienen un rango de diferencia aproximado de $0.5 \%,{ }^{26-28}$ que es menor que la población general, ya que los individuos que acuden a donar generalmente se consideran sanos.

\section{CONCLUSIONES}

La donación de tipo reposición (familiar) sobrepasa por mucho a la altruista y, de esta manera, aquélla es la mayor fuente de hemoderivados recibida en este banco de sangre.

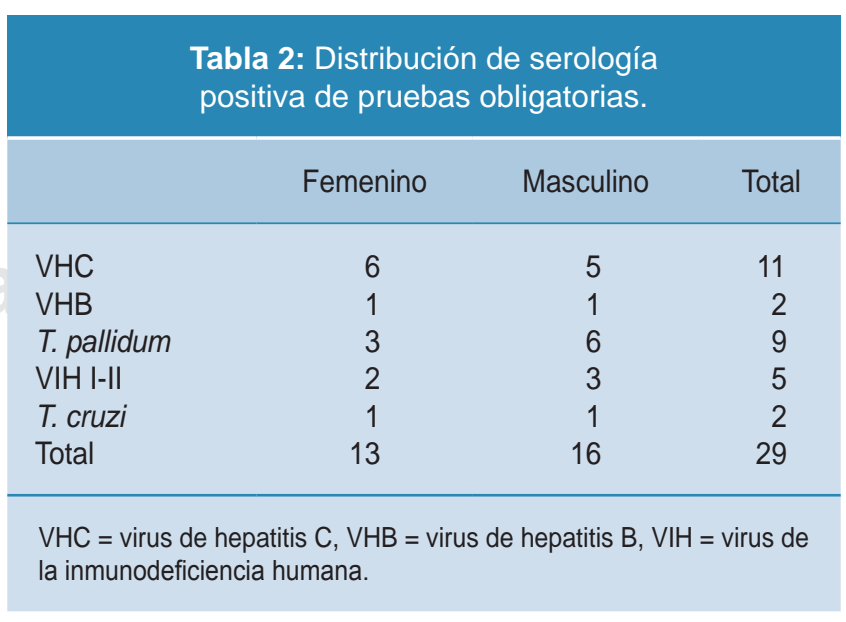


El tamizaje clínico inicial es de suma importancia, pues las nuevas tecnologías altamente sensibles y específicas contribuyen a este tamizaje.

En nuestro estudio se encontró que la prevalencia de infección por $\mathrm{VHC}$ en donadores tiene una distribución heterogénea. Es de relevancia que el grupo más afectado fuera el de mujeres entre 45 a 65 años. A pesar de esto, la frecuencia demostrada fue de $0.27 \%$ de la población total. La prevalencia de infección por VHB fue aún más baja con $0.04 \%$ sin diferencias entre género y/o edad. La detección de VHC fue 5.5 veces más prevalente que la de VHB. Se considera que esta baja prevalencia pudo deberse a que la mayoría de los individuos que acuden a donar sangre, en términos generales, se encuentran en buenas condiciones de salud.

\section{REFERENCIAS}

1. Marrón-Peña M. Historia de la transfusión sanguínea. Rev Mex Anest. 2017; 40: 233-238.

2. Kim MJ, Park Q, Min HK, Kim HO. Residual risk of transfusiontransmitted infection with human immunodeficiency virus, hepatitis $C$ virus, and hepatitis B virus in Korea from 2000 through 2010. BMC Infect Dis. 2012; 12: 160. doi: 10.1186/ 1471-2334-12-160.

3. Schweitzer A, Horn J, Mikolajczyk RT, Krause G, Ott JJ. Estimations of worldwide prevalence of chronic hepatitis B virus infection: a systematic review of data published between 1965 and 2013. Lancet. 2015; 386 (10003): 1546-1555.

4. World Health Organization. Global hepatitis report. Geneva: World Health Organization; 2017.

5. Calleja PJ, Llop HE, Ruiz MM, De la Revilla NJ, Calvo BE, Pons RF et al. Prevalence of viral hepatitis (Band C) serological warkers in healthy working population. Rev Esp Enferm Dig. 2013; 105: 249-254.

6. Cobb B, Pockros PJ, Vilchez RA, Vierling JM. HCV RNA viral load assessments in the era of direct-acting antivirals. Am J Gastroenterol. 2013; 108: 471-475.

7. World Health Organization. Hepatitis B. Fact Sheet $N^{\circ}$ 204. [sede web]. [actualizada 27 julio 2020] Disponible en: http://www.who. int/mediacentre/factsheets/fs204/en/.

8. Trépo C, Chan HL, Lok A. Hepatitis B virus infection. Lancet. 2014; 384: 2053-2063.

9. Sanchez LV, Maldonado M, Bastidas-Ramirez BE, Norder H, Panduro A. Genotypes and S-gene variability of Mexican hepatitis B virus strains. J Med Virol. 2002; 68: 24-32.

10. World Health Organization. Introduction of hepatitis $B$ vaccine into childhood immunization services: management guidelines, including information for health workers and parents [sede web]. Geneva: WHO, Department of Vaccines and Biologicals; 2001. Disponible en: https://apps.who.int/iris/bitstream/handle/10665/66957/ WHO_V-B_01.31_eng.pdf?sequence $=1$ \&isAllowed $=y$
11. Panduro A, Roman S, Khan A, Tanaka Y, Kurbanov F, Martinez-Lopez $\mathrm{E}$ et al. Molecular epidemiology of hepatitis $\mathrm{C}$ virus genotypes in West Mexico. Virus Research. 2010; 151: 19-25.

12. Jimenez-Mendez R, Uribe-Salas F, Lopez-Guillen P, Cisneros-Garza L, Castañeda-Hernandez G. Distribution of HCV genotypes and HCV RNA viral load in different regions of Mexico. Ann Hepatol. 2010; 9: 33-39.

13. Gardenier D, Kwong J, Olson M, Epstein R. Epidemiology, screening, and pretreatment evaluation of the patient with chronic hepatitis $\mathrm{C}$ infection. J Nurse Pract. 2015; 11: 109-115.

14. Halabe ChJ, Angulo VF. Hepatitis viral. Rev Fac Med UNAM. 2000; 43: $90-100$

15. Rivero-Jiménez RA. Transmisión de infecciones virales por la transfusión de sangre. Rev Cubana Hematol Inmunol Hemoter. 2006; 22.

16. World Health Organization. Los datos más recientes ponen de relieve la necesidad de actuar urgentemente a nivel mundial contra las hepatitis [sede web]. Ginebra: Organización Mundial de la Salud. [actualizado 21 Abril 2017]. Disponible en: https://www.who.int/es/ news-room/detail/21-04-2017-new-hepatitis-data-highlight-needfor-urgent-global-response.

17. Secretaría de Salud. Anuario de morbilidad 1984-2015. Estados Unidos Mexicanos: SUIVE/DGE Secretaría de Salud; 2015.

18. Abbott. Manual de operaciones del sistema Architect [sede web]. Abbott Laboratories. 2013.

19. Abbott. Manual de operaciones del sistema Architect [sede web]. Abbott Laboratories Anti HCV. 2014.

20. Abbott. Manual de operaciones del sistema Architect [sede web]. Abbott Laboratories HBsAg Qualitative II. 2013.

21. Abbott. Manual de operaciones del sistema Architect [sede web]. Abbott Laboratories Anti-HBc II. 2015.

22. Hernández-Pérez RE, Frías-Salcedo JA, Del Ángel-Guevara O. Seroprevalencia de anticuerpos contra el virus de la hepatitis $C$ en donadores de sangre del Hospital Central Militar. Salud Publica Mex. 1994; 36: 538-540.

23. Álvarez-Muñoz MT, Bustamante-Calvillo ME. Hepatitis B y Delta: prevalencia de marcadores cero epidemiológicos en donadores de sangre voluntarios y su grupo familiar. Gac Med Mex. 1991; 127: 399-404.

24. Méndez-Sánchez N, Baptista-González H, Sánchez-Gómez RH, Bordes-Aznar J, Uribe-Esquivel M. Prevalencia de hepatitis B y C en un Hospital de Tercer Nivel de la Ciudad de México. Salud Pública de México. 1999; 41: 6.

25. Organización Panamericana de la Salud. Suministro de sangre para transfusiones en los países de Latinoamérica del Caribe. OPS; 2017.

26. Rivera-López MRF, Zavala-Méndez C, Arenas-Esqueda A. Prevalencia de seropositividad para $\mathrm{VIH}$, hepatitis B y $\mathrm{C}$ en donadores de sangre. Gac Méd Méx. 2004; 140 (6): 657-660.

27. Souto-Meiriño CA, Simón-Domínguez J, Pulido-Priego MA, Hernández-Pérez A, García-Hernández IC, Del Río-Chiriboga CA. Prevalencia de marcadores para hepatitis A, B y C en un hospital de México. Salud Publica de México. 1994; 36 (3): 257-262.

28. Carreto-Vélez MA, Carrada-Bravo T, Martínez-Magdaleno A. Seroprevalencia de $\mathrm{VHB}, \mathrm{VHC}$ y $\mathrm{VIH}$ en donadores de sangre en Irapuato, México. Salud Pública de México. 2003; 45 (S5): 690-693.

Conflicto de intereses y financiamiento: Ninguno. 